\title{
MedienPädagogik
}

Zeitschrift für Theorie und Praxis der Medienbildung

Themenheft Nr. 28: Tagungsband: Bildung gemeinsam verändern: Diskussionsbeiträge und Impulse aus Forschung und Praxis. Herausgegeben von David Meinhard, Valentin Dander, Andrea Gumpert, Christoph Rensing, Klaus Rummler und Timo van Treeck.

\section{Empirische Ergebnisse zur Nutzung sozialer Netzwerkplattformen durch E-Learning- Akteurinnen und Akteure an Hochschulen}

Philip Meyer

\begin{abstract}
Zusammenfassung
Das Informationsportal "e-teaching.org» richtet sich an E-Learning-Akteure/-innen an Hochschulen, denen es Bildungsinhalte und aktuelle Informationen zu didaktischen, technologischen und organisatorischen Aspekten des Lernens mit digitalen Medien bietet. In den Monaten Juli und August 2014 nahmen 137 Nutzer/innen an einer halb-standardisierten, nicht-repräsentativen Online-Befragung teil, welche die Bedeutung sozialer Netzwerke für das Portal in Hinblick auf Austausch- und Informationsprozesse in beruflichen und privaten Kontexten erhob. Zudem wurde das Twitternetzwerk des Portals mit rund 40.000 Verbindungen zwischen 1.600 Personen (Stand: April 2014) anhand einer sozialen Netzwerkanalyse untersucht. Es deutet sich an, dass soziale Netzwerke zu bestimmten Zwecken professionell genutzt werden. Bei der Mehrzahl der Befragten ist dies auf Twitter die gegenseitige Vernetzung sowie die Informationsaufnahme und -streuung, wohingegen auf Facebook die Teilnahme und der soziale Austausch in Gruppen dominiert. Mit rund einem Drittel sieht allerdings ein nicht unerheblicher Teil der Befragten von einer beruflichen Beteiligung in sozialen Netzwerken ab und nutzt stattdessen lieber E-Mails, Blogs und RSS-Feeds.
\end{abstract}

Use of Social Network Platforms by E-Learning Professionals in Higher Education: an Empirical Study

\begin{abstract}
The non-commercial information portal «e-teaching.org» addresses professionals in technology-supported learning in higher education institutions. It publishes educational content and frequent news regarding didactical, technological and organisational aspects of learning with digital media. In July and August 2014, 137 portal users participated in a semi-standardised, non-representative survey, which collected data on the usage behavior and on the perceived importance of social networks for communication and information processes in professional and personal contexts. In addition, the Twitter network of e-teaching.org, which contains about 40.000 links between 1.600 persons (as of April 2014), was investigated through social network analysis methodology. Results
\end{abstract}


suggest that social networks are used professionally for particular purposes. On Twitter, for a majority, networking and the collection and dissemination of information are of most importance, while on Facebook the participation and discussion in groups seems to be the dominant motive. Approximately one third of e-learning professionals are not using social media much for their job, rather relying on e-mails, blogs and RSS feeds.

\section{Kontext der Erhebung}

Soziale Netzwerke nehmen für die Informationsaufnahme und den niedrigschwelligen Austausch mittlerweile einen hohen Stellenwert ein. Während der Austausch mit Freunden und Familienangehörigen dort seit jeher die Hauptmotivation darstellt, verwenden inzwischen $22 \%$ aller Internetnutzenden soziale Netzwerke auch, um sich über das aktuelle Tagesgeschehen zu informieren (Bitkom Research 2015). Es liegt die Vermutung nahe, dass auch im Beruf eine stärkere Nutzung sozialer Netzwerke stattfindet. In diesem Beitrag ist ganz speziell von Interesse, welche Rolle soziale Netzwerke für Personen spielen, die mit dem Portal «e-teaching.org» in Kontakt stehen und in der Regel in der E-Learning-Qualifizierung an Hochschulen tätig sind. Das Portal richtet sich seit Anfang der 2000er Jahre an Hochschullehrende und hochschuldidaktische Multiplikatoren/-innen, denen es Bildungsinhalte und Informationen zu didaktischen, technologischen und organisatorischen Aspekten des ELearnings im Anwendungskontext Hochschule bereitstellt (Thillosen 2011). Klassische Kommunikationskanäle, die das Portal in der Vergangenheit nutzte, waren die E-Mail in Verbindung mit einem PDF-Magazin und ein per RSS abonnierbarer Blog. Um Erkenntnisse darüber zu erhalten, welche Bedeutung soziale Netzwerke mittlerweile für die Arbeit von Hochschulakteuren/-innen im Feld des technologiebasierten Lernens spielen, wurden im Rahmen des Forschungs- und Entwicklungsprojekts e-teaching.org im Kontext sozialer Netzwerke» (Meyer o. J.) eine Online-Umfrage und eine soziale Netzwerkanalyse nach Jansen (vgl. 2006) durchgeführt, deren Ergebnisse im Folgenden dargelegt werden.

\section{Ziel und Methodik der Befragung}

Ziel der Erhebung war der Gewinn von Erkenntnissen zur Nutzung sozialer Netzwerkplattformen durch E-Learning-Akteure/-innen an Hochschulen, um die Redaktionsarbeit von e-teaching.org dahingehend auszurichten. In den Monaten Juli und August 2014 wurde hierfür eine nicht-repräsentative Online-Befragung im deutschsprachigen Raum durchgeführt. Basis waren Nutzende von e-teaching.org, wobei insgesamt 137 Personen über den Newsletter und die Webseite des Portals rekrutiert werden konnten. Für die sozialen Netzwerke Facebook, Twitter, Google+, Xing und LinkedIn wurde zunächst mit Likert-Skalen nach der allgemeinen und kontextspezifischen 
Nutzungsintensität gefragt («Wie häufig nutzen Sie [Soziales Netzwerk] für private/ berufliche/lehrbezogene Zwecke?»). Des Weiteren interessierte die Verteilung der Nutzungsmotive «Vernetzung», «Diskussion», «Information» und «Verbreitung» von Beiträgen (unterteilt in Eigen- und Fremdbeiträge). Auch das über die Netzwerke hinausgehende Informationsverhalten wurde abgefragt, wobei Freitextangaben möglich waren. Die restlichen Fragen bezogen sich auf die Evaluation von e-teaching.org. Hier ging es um Themenpräferenzen sowie die Meinung zum Portal - allgemein und bezogen auf die sozialen Medien. Für die Auswertung wurde auf deskriptive statistische Verfahren zurückgegriffen, wobei aufgrund der kleinen Stichprobe keine Berechnung von Signifikanzwerten erfolgte.

\section{Ergebnisse der Befragung}

Die Ergebnisse der Befragung geben zunächst Einblick in die grundsätzliche Demografie der e-teaching.org-Nutzenden. Im Anschluss werden die Ergebnisse zur beruflichen und privaten Nutzung sozialer Netzwerke berichtet.

\section{Demografie}

Die Befragten lassen sich mit grosser Mehrheit (73\%) in die Zielgruppe der Akteure/innen an Hochschulen einordnen. 84\% sind oder waren in der (nicht nur universitären) Lehre tätig, besonders häufig in den Fächern Pädagogik und Didaktik (37\%) oder Informatik (19\%). 67\% gehören dem akademischen Mittelbau an; 36\% haben einen Doktorgrad oder höheren akademischen Titel. $60 \%$ sind weiblich; $67 \%$ im Alter zwischen 30 und 50 Jahren.

\section{Nutzung sozialer Netzwerke}

$82 \%$ der Befragten sind zumindest «selten» in sozialen Medien aktiv. In Bezug auf die Aktivität gibt es grosse Unterschiede zwischen den Netzwerken: «Sehr häufig (fast täglich/täglich)» nutzen den Umfrageergebnissen zufolge 37\% Facebook, 21\% Twitter, $7 \%$ Xing und $6 \%$ Google+ (siehe Abb. 1), wobei sich auf Facebook vorwiegend jüngere Personen finden: $64 \%$ sind unter 40 Jahre alt. Die Befragten haben dabei häufig eine Präferenz für ein bestimmtes soziales Netzwerk: $24 \%$ sind nur bei Facebook registriert, 14\% ausschliesslich bei Twitter. Insgesamt liegt die Nutzung der Netzwerke deutlich über dem Durchschnitt der deutschen Internetnutzer/innen, von denen laut ARD/ZDF-Onlinestudie täglich 22\% Facebook und 1\% Twitter nutzen (Tippelt \& Kupferschmitt 2015, 443). 


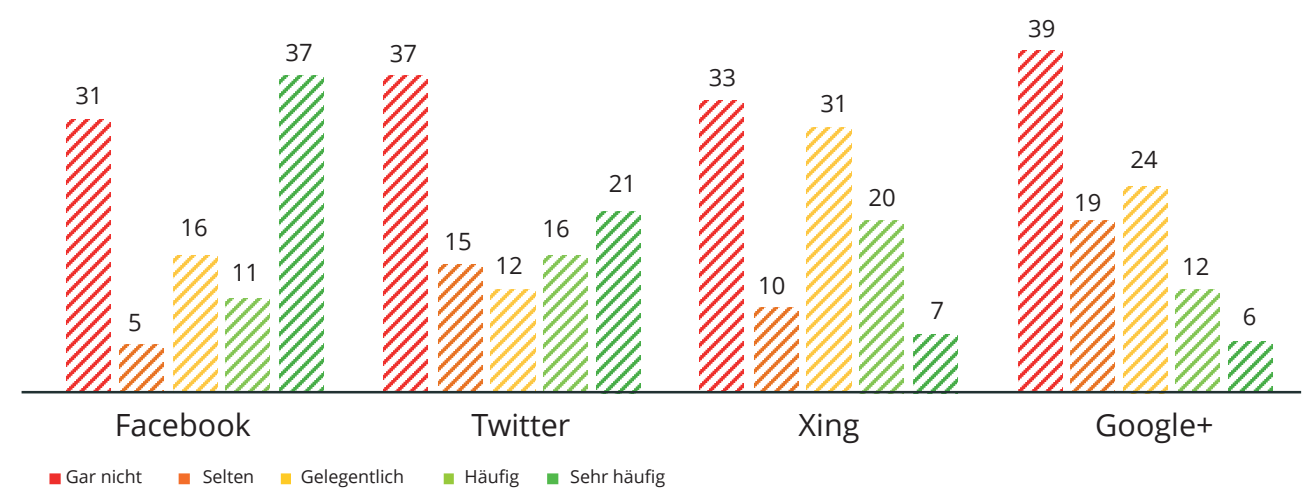

Abb. 1.: Gesamtnutzung verschiedener sozialer Netzwerke in Prozent $(n=137)$.

\section{Berufliche Nutzung}

Twitter wird von $62 \%(n=74)$ der Nutzenden auch beruflich verwendet (mind. «stimme eher zu»), Facebook von 53\% ( $n=85$; siehe Abb. 2). In der Lehre finden die beiden sozialen Netzwerke seltener Anwendung: 18\% der Facebook-User geben eine Nutzung an, von den Twitter-Usern gut ein Viertel (28\%). Ein grundsätzliches Themeninteresse für den Einsatz von «Web 2.0 in der Lehre» ist bei 42\% aller Akteure/-innen vorhanden.

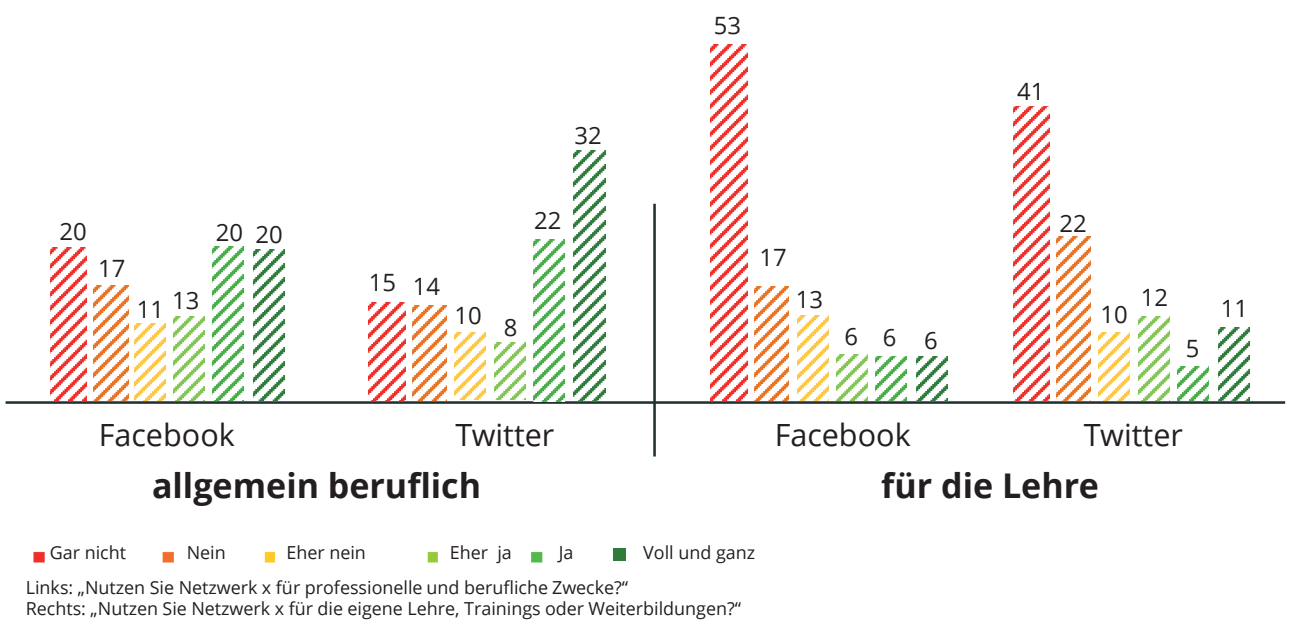

Abb. 2.: Berufliche Nutzung von Facebook $(n=85)$ und Twitter $(n=74)$ in Prozent.

Facebook wird beruflich vor allem genutzt, um sich dort an Gruppen zu beteiligen (39\%). Twitter wird primär für die Information über E-Learning-Themen verwendet. Für $70 \%$ ist dies die Motivation neben der Vernetzung mit Kollegen/-innen. Letztgenanntes Motiv ist mit je 31\% sowohl für Facebook-als auch für Twitter-Nutzer/innen von Bedeutung. 


\section{Soziale Netzwerkanalyse}

In die soziale Netzwerkanalyse (SNA) nach Jansen (2006) wurden Akteure/-innen einbezogen, die e-teaching.org auf Twitter folgen. Die Auswertung geografischer Angaben (siehe Abb. 3) ergibt ein Bild der interregionalen Verbindungen. Die Achse zwischen Stuttgart und dem Ruhrgebiet ist besonders dicht vernetzt. Die Helligkeit der Linien lässt auf die Popularität der Nutzer/innen schliessen: Verbindungen zwischen Akteuren/-innen mit vielen Followern werden heller dargestellt. Die Schriftgrösse der Städtenamen deutet an, wie viele E-Learning-Akteure/-innen dem Portal am jeweiligen Standort auf Twitter folgen, wobei kleinere Standorte ausgeblendet wurden. Vor allem die Grossstädte Berlin und Hamburg sind hier deutlich sichtbar.

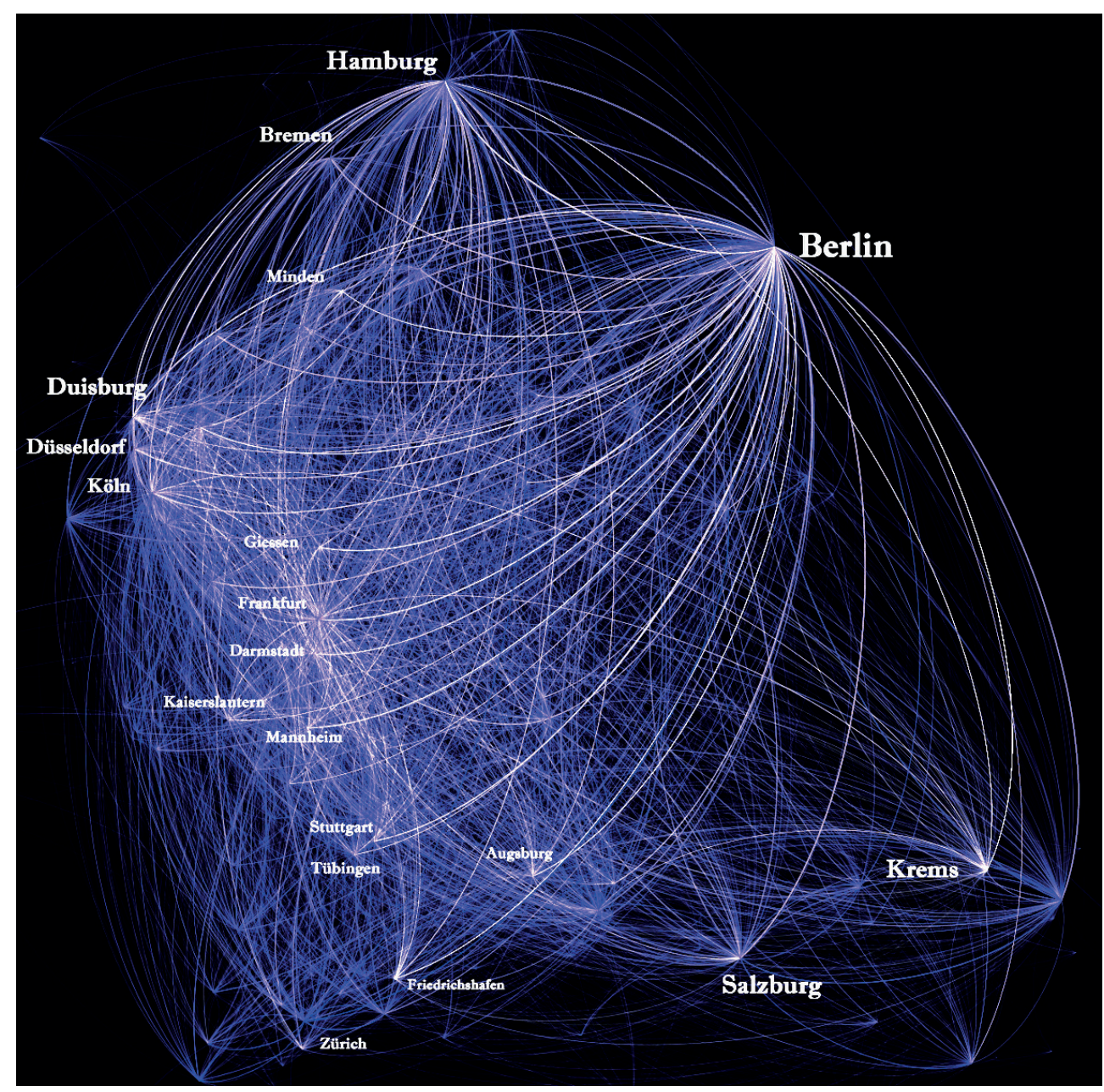

Abb. 3.: Regionale Vernetzung von E-Learning-Akteuren/-innen (Basis: Twitter, $\mathrm{N}=1.639$, $\mathrm{n}=41.519)$. 
Die Accounts liessen sich anhand ihrer Vernetzungsstärke, d. h. des Folgens und Gefolgt-Werdens untereinander (Beidseitigkeit wurde doppelt gewichtet), hierarchisch in drei ähnlich grosse Cluster einteilen. Eine manuelle inhaltliche Prüfung der Cluster anhand der Twitter-Profilbeschreibungen ergab im ersten Cluster überwiegend Personen an Lehrstühlen und E-Learning-Einrichtungen im Hochschulbereich, im zweiten Cluster Personen an Schulen und in der Lehrerbildung sowie im dritten Cluster überregionale Institutionen (wie z. B. der Deutsche Bildungsserver, der Stifterverband oder das Portal «Lehrer Online»).

\section{Grenzen der Untersuchung}

Die Studie kann aufgrund der Fokussierung der besonders nutzerstarken sozialen Netzwerke keine Aussagen über die allgemeine Nutzung der vielfältigen verfügbaren Social-Media-Dienste durch die Akteure/-innen treffen. Die Gruppe der e-teaching. org-Nutzer/innen (Befragung) bzw. Follower (SNA) repräsentiert zudem nicht notwendigerweise die Grundgesamtheit aller E-Learning-Akteure/-innen und Selektionseffekte sind aufgrund der verhältnismässig kleinen Stichprobe zu erwarten.

\section{Schlussfolgerungen}

Soziale Netzwerke bieten zahlreiche Chancen für die Kommunikation von Informationsportalen wie e-teaching.org. Angesichts des Resultats, dass Facebook mittlerweile auch für die Beteiligung an beruflich ausgerichteten Gruppen eine wichtige Rolle spielt und sich dort viele Personen finden, die über Twitter nicht erreicht werden, erscheint es sinnvoll, dort präsent zu sein, indem man sich an Gruppen beteiligt oder eigene Seiten und Gruppen eröffnet. Über Twitter werden - so die zentrale Erkenntnis der Netzwerkanalysen - Akteure erreicht, die nicht nur dem engeren Kreis der Zielgruppe zuzuordnen sind, die sich im Fall von e-teaching.org mit E-Learning an Hochschulen beschäftigt. Vielmehr entsteht dort ein öffentliches Forum, das die Möglichkeit bietet, für die eigenen Themen eine hohe Reichweite abseits fester Gruppenstrukturen zu bekommen. Durch die häufig eingeschränkten Sichtbarkeitseinstellungen ist dies bei Facebook weniger der Fall. Es empfiehlt sich daher, mehrere Netzwerkplattformen für die Kommunikation von Informationsportalen zu verwenden. Nichtsdestotrotz sind selbst bei der internetaffinen Zielgruppe von e-teaching. org knapp 20\% der befragten Personen gar nicht über soziale Netzwerke erreichbar, weshalb auch einem E-Mail-Newsletter als Kommunikationskanal nachwievor eine hohe Bedeutung zukommt.

Die Ergebnisse wurden primär zur Weiterentwicklung der Kommunikation des Portals e-teaching.org genutzt. Auch eine verstärkte Einbindung der Community über 
Umfragen und Gruppendiskussionen in den sozialen Netzwerken wurde in der Folge realisiert (vgl. Meyer 2014).

\section{Literatur}

Bitkom Research. 2015. «Umfrage zur Nutzung von sozialen Netzwerken als Nachrichtenquelle in Deutschland.» Presseinformation. https://www.bitkom.org/Presse/Presseinformation/ Jeder-Fuenfte-nutzt-soziale-Netzwerke-als-Nachrichtenquelle.html

Jansen, Dorothea. 2006. Einführung in die Netzwerkanalyse. Grundlagen, Methoden, Forschungsbeispiele. Wiesbaden: VS/GWV.

Meyer, Philip. 2014. «Erste Projektergebnisse: e-teaching.org im Kontext sozialer Netzwerke.» In Newsletter e-teaching.org, herausgegeben von Anne Thillosen, Markus Schmidt und Philip Meyer, 36:2-3. https://www.e-teaching.org/news/letter/Newsletter-36_Oktober-2014_l. pdf.

Meyer, Philip. o. J. «e-teaching.org im Kontext sozialer Netzwerke. Projektbeschreibung auf der Webseite des Leibniz-Instituts für Wissensmedien». https://www.iwm-tuebingen.de/ www/de/forschung/projekte/projekt.html?name=E-TeachingSozialeNetzwerke.

Thillosen, Anne. 2011. «e-teaching.org: ein Informationsportal zur Unterstützung von E-Learning an deutschen Hochschulen. Rückblick, Erfahrungen und Perspektiven.» Ludwigsburger Beiträge zur Medienpädagogik, 14, 1-5.

Tippelt, Florian, und Thomas Kupferschmitt. 2015. «Social Web: Ausdifferenzierung der Nutzung - Potenziale für Medienanbieter. Ergebnisse der ARD/ZDF-Onlinestudie 2015.» Media Perspektiven, 10/2015, 442-452.

\section{Abbildungen}

Abb. 1.: Gesamtnutzung verschiedener sozialer Netzwerke in Prozent $(\mathrm{n}=137)$.

Abb. 2.: Berufliche Nutzung von Facebook $(n=85)$ und Twitter $(n=74)$ in Prozent.

Abb. 3.: Regionale Vernetzung von E-Learning-Akteuren/-innen (Basis: Twitter, $\mathrm{N}=1.639$, $\mathrm{n}=41.519)$. 